\title{
MLN8237 treatment in an orthoxenograft murine model for malignant peripheral nerve sheath tumors
}

\author{
*Russell Payne, MD, Oliver D. Mrowczynski, PhD, Becky Slagle-Webb, BS, Alexandre Bourcier, \\ Christine Mau, MD, Dawit Aregawi, MD, Achuthamangalam B. Madhankumar, PhD, \\ Sang Y. Lee, PhD, Kimberly Harbaugh, MD, James Connor, PhD, and Elias B. Rizk, MD, MSc
}

Department of Neurosurgery, Penn State Milton S. Hershey Medical Center, Hershey, Pennsylvania

\begin{abstract}
OBJECTIVE Malignant peripheral nerve sheath tumors (MPNSTs) are soft-tissue sarcomas arising from peripheral nerves. MPNSTs have increased expression of the oncogene aurora kinase A, leading to enhanced cellular proliferation. This makes them extremely aggressive with high potential for metastasis and a devastating prognosis; 5 -year survival estimates range from a dismal $15 \%$ to $60 \%$. MPNSTs are currently treated with resection (sometimes requiring limb amputation) in combination with chemoradiation, both of which demonstrate limited effectiveness. The authors present the results of immunohistochemical, in vitro, and in vivo analyses of MLN8237 for the treatment of MPNSTs in an orthoxenograft murine model.
\end{abstract}

METHODS Immunohistochemistry was performed on tumor sections to confirm the increased expression of aurora kinase A. Cytotoxicity analysis was then performed on an MPNST cell line (STS26T) to assess the efficacy of MLN8237 in vitro. A murine orthoxenograft MPNST model transfected to express luciferase was then developed to assess the efficacy of aurora kinase A inhibition in the treatment of MPNSTs in vivo. Mice with confirmed tumor on in vivo imaging were divided into 3 groups: 1) controls, 2) mice treated with MLN8237, and 3) mice treated with doxorubicin/ifosfamide. Treatment was carried out for 32 days, with imaging performed at weekly intervals until postinjection day 42 . Average bioluminescence among groups was compared at weekly intervals using 1-way ANOVA. A survival analysis was performed using Kaplan-Meier curves.

RESULTS Immunohistochemical analysis showed robust expression of aurora kinase A in tumor cells. Cytotoxicity analysis revealed STS26T susceptibility to MLN8237 in vitro. The group receiving treatment with MLN8237 showed a statistically significant difference in tumor size compared with the control group starting at postinjection day 21 and persisting until the end of the study. The MLN8237 group also showed decreased tumor size compared with the doxorubicin/ifosfamide group at the conclusion of the study $(p=0.036)$. Survival analysis revealed a significantly increased median survival in the MLN8237 group ( 83 days) compared with both the control ( 64 days) and doxorubicin/ifosfamide (67 days) groups. A hazard ratio comparing the 2 treatment groups showed a decreased hazard rate in the MLN8237 group compared with the doxorubicin/ifosfamide group (HR 2.945; $p=0.0134$ ).

CONCLUSIONS The results of this study demonstrate that MLN8237 is superior to combination treatment with doxorubicin/ifosfamide in a preclinical orthoxenograft murine model. These data have major implications for the future of MPNST research by providing a robust murine model as well as providing evidence that MLN8237 may be an effective treatment for MPNSTs.

https://thejns.org/doi/abs/10.3171/2017.8.JNS17765

KEY WORDS alisertib; malignant peripheral nerve sheath tumor; MPNST; neurofibromatosis; MLN8237; aurora kinase; oncology; small-molecule inhibitor

\footnotetext{
ABBREVIATIONS FBS = fetal bovine serum; IP = intraperitoneal; IVIS = intravital imaging spectroscopy; MPNST = malignant peripheral nerve sheath tumor; NF1 = neurofibromatosis type 1; PBS = phosphate-buffered saline.

SUBMITTED March 26, 2017. ACCEPTED August 1, 2017.

INCLUDE WHEN CITING Published online February 23, 2018; DOI: 10.3171/2017.8.JNS17765.

${ }^{*}$ R.P. and O.D.M. contributed equally to this work.
} 
$\mathrm{M}$ ALIGNANT peripheral nerve sheath tumors (MPNSTs) are a rare type of soft-tissue sarcoma that arise from peripheral nerves. ${ }^{17,41}$ They most often occur in the extremities and in large nerve trunks, such as the sciatic nerve. ${ }^{23,47}$ These tumors comprise $2 \%$ of all sarcomas and approximately 5\%-10\% of the 6000 soft-tissue sarcomas diagnosed each year. ${ }^{18,35,52}$ They can arise sporadically or after radiation therapy, and approximately one-half occur in the setting of neurofibromatosis type 1 (NF1). ${ }^{13-15,31}$ Several MPNST cell lines have increased expression of the oncogene aurora kinase A, which is thought to lead to enhanced cellular proliferation. MPNSTs are extremely aggressive, with high potential for metastasis and a dismal 15\%-60\% 5-year survival rate. . $^{13-15,29,30}$

Complete resection remains the mainstay of treatment and has been shown to improve survival. ${ }^{4,6,12,43,48,51}$ Radiotherapy has been found to be beneficial for local control and to delay recurrence but does not improve long-term survival. ${ }^{17}$ MPNSTs, like most soft-tissue sarcomas, are insensitive to chemotherapy, and chemotherapy alone has not proven to be effective. ${ }^{55}$ Although there is no consensus on the optimal chemotherapeutic regimen, doxorubicin-based multiagent therapy with ifosfamide has been used with local control, with some modest success. ${ }^{6,26,40}$, 53,55 Standard of care therefore consists of resection of the lesion with a combination of adjuvant radiation and chemotherapy. Unfortunately, complete resection is often not possible due to tumors arising in inoperable locations or having already metastasized. ${ }^{1,4,53}$ This highlights the importance of developing safe and effective systemic therapies with low side-effect profiles.

One potential therapy is MLN8237 (alisertib), an orally bioavailable aurora kinase A inhibitor that has been found to have activity in several types of solid tumors, including MPNSTs. ${ }^{28,34,36}$ Inhibiting aurora kinase blocks its actions on centrosome maturation and chromosome separation during metaphase, leading to mitotic spindle defects, aneuploidy, and ultimately, cell death. , $29,30^{2}$

We present the results of a preclinical study, which used an orthoxenograft non-NF1 MPNST murine model that we developed. In this study, the efficacy of oral MLN8237 monotherapy was compared with gold standard combination therapy of intravenous doxorubicin and ifosfamide. Together, our data have major implications for the future of MPNST research by providing a robust murine model as well as evidence that MLN8237 is an effective treatment for MPNSTs.

\section{Methods}

\section{Cell Line Characteristics and Preparation}

\section{Cell Line Characteristics}

The STS26T sarcoma cell line, which is a human MPNST cell line, was generously gifted by Dr. Daniel Scoles, University of Utah. The STS26T cell line has previously been validated and characterized as a human nonNF1 MPNST cell line. ${ }^{11,16,32,37,42,54}$ All of the cell lines were cultured in DMEM (Gibco) supplemented with $10 \%$ fetal bovine serum (FBS). The cell line was maintained at $37^{\circ} \mathrm{C}$ in a humidified incubator with $5 \% \mathrm{CO}_{2}$.

\section{STS26T Cell Line Luciferase Transfection}

In order for the cells to express the luciferase vector for bioluminescent imaging through intravital imaging spectroscopy (IVIS), the STS26T cell line was transfected with vector pGL4.51/luc2/CMV/Hygro from Promega. To initially establish a stably transfected cell line, the cytotoxicity of the hygromycin was determined in the STS26T cells. Subsequently, STS26T cells were treated with a lipofectamine and plasmid mixture in the ratio according to the manufacturer's instructions (Promega). The complex was then added to the cells and allowed to transfect. The transfection process caused death of some cells. However, after 24 hours, fresh medium (DMEM with $10 \%$ FBS) with hygromycin was added, and the cells were allowed to grow for a few days until colonies were formed. Subsequently, a few colonies were selected and clonogenic rings were placed over the colonies. Of the several colonies chosen, only 1 colony grew and amplified in the presence of hygromycin. The presence of the luciferase vector in the cells was then confirmed by treating the cells with XenoLight Luciferin-D substrate (Caliper LS) and exposing the tubes to a chemiluminescence imager, where dark color was seen in the transfected cells. Further confirmation was obtained after injecting them into the murine model.

\section{Cell Culture and Preparation for Tumor Cell Injection}

The STS26T cell line was cultured with complete DMEM media supplemented with 10\% FBS until 80\%$90 \%$ confluency. Cells were then washed with $10 \mathrm{ml}$ of phosphate-buffered saline (PBS; Corning), and 0.5\% trypsin-EDTA 10× (Gibco by Life Technologies) was diluted to $1 \times$ with PBS. One milliliter of $1 \times$ trypsin was then incubated with the cells at $37^{\circ} \mathrm{C}$ for 1 minute to detach the cells from the culture flask. The cells were resuspended in DMEM media with $10 \%$ FBS and centrifuged at $2400 g$ (IEC Centra CL2) for 2.5 minutes. The media was aspirated, and the cells were washed again with media and centrifuged at $2400 \mathrm{~g}$ for 2.5 minutes. The media was aspirated again. The cells were subsequently resuspended in $1 \mathrm{ml}$ of media and counted manually. The cells were then diluted to 50,000 cells $/ 5 \mu \mathrm{l}$ of media. This cell resuspension was then aspirated into a 30 -gauge Hamilton syringe and used for injection into the mouse sciatic nerve.

\section{Immunohistochemistry of Cells in Culture and Tumor Tissue Sections}

To analyze aurora kinase A expression in STS26T cells, cells were plated on chamber slides at a density of $20,000 /$ spot in a volume of $25 \mu \mathrm{l}$ and allowed to attach for 24 hours. The cells were then washed with PBS, fixed with $4 \%$ paraformaldehyde for 20 minutes, and again washed with PBS for 5 minutes. The nonspecific binding sites on the cells were blocked with $10 \%$ normal goat serum for 30 minutes at $37^{\circ} \mathrm{C}$ in an incubator. The cells were subsequently exposed to anti-AurkA (1:200 sc-373856) antibody overnight at $4^{\circ} \mathrm{C}$ and washed with PBS 3 times for 5 minutes each wash. Next, the slides were treated with DAPI and secondary Alexa Fluor 488-conjugated antimouse $\operatorname{IgG}$ for 60 minutes and then washed with PBS 3 
times before gel mounting and viewing under a fluorescence microscope (Nikon Eclipse 80i).

To analyze expression of tumor proliferation, cell cycle, and apoptosis proteins after treatment, tumor tissue was obtained from mice in each treatment group and immunohistochemistry was performed. The tumor tissues were frozen and sectioned to $10 \mu \mathrm{m}$ using a cryostat and subjected to immunohistochemistry to identify protein expression. To perform this study, sections were fixed with 4\% paraformaldehyde for 20 minutes and washed with PBS for 5 minutes. The nonspecific binding sites on the tissues were blocked with $10 \%$ normal goat serum for 30 minutes at $37^{\circ} \mathrm{C}$ in an incubator. Subsequently, tumor tissues were incubated with Ki-67 antibody (1:500 ab15580), cleaved caspase-3 (1:500 9661S), aurora kinase A (1:200 sc-373856), or cyclin B1 (1:200 sc-752) overnight at $4{ }^{\circ} \mathrm{C}$ and then washed with PBS 3 times for 5 minutes each wash. The sections were treated with DAPI and secondary Alexa Fluor 488-conjugated anti-rabbit IgG for $60 \mathrm{~min}$ utes and then washed with PBS 3 times before gel mounting and viewing under a fluorescence microscope.

\section{In Vitro Cytotoxicity Analysis}

To assess the cytotoxicity of MLN8237 in the cell culture model, the STS26T MPNST cells were cultured in 96-well plates at a concentration of 4000 cells per well for 24 hours. After the 24-hour incubation period, cells were treated with MLN8237 solubilized in dimethyl sulfoxide diluted in DMEM media at concentrations of $0,1,10$, 100 , and $1000 \mathrm{nM}$, and incubated for 48 hours. The cells were then treated with $20 \mu \mathrm{l}$ of 3-(4,5-dimethylthiazol2-yl)-5-(3-carboxymethoxyphenyl)-2-(4-sulfophenyl)-2Htetrazolium (Promega) according to the manufacturer's instructions. After 4-6 hours of incubation, the plate absorbance was recorded at $490 \mathrm{~nm}$ using a 96-well plate reader. The measurement was made in quintuplicate, and the percentage control was measured with respect to the untreated cells.

\section{Animal Model}

We first anesthetized 42 NIH III nude mice from Charles River Laboratories using a concentration of 4\%$5 \%$ isoflurane within an induction chamber. Mice were then transferred onto the working surface, and anesthesia was maintained using an isoflurane (1\%-2\%)-emitting nose cone. The procedure was then performed as depicted in Fig. 1A-E.

\section{Imaging of Nerve Tumors}

Three days after injection of tumor cells, mice were imaged using IVIS. Mice were first anesthetized in an induction chamber using a concentration of $4 \%-5 \%$ isoflurane.
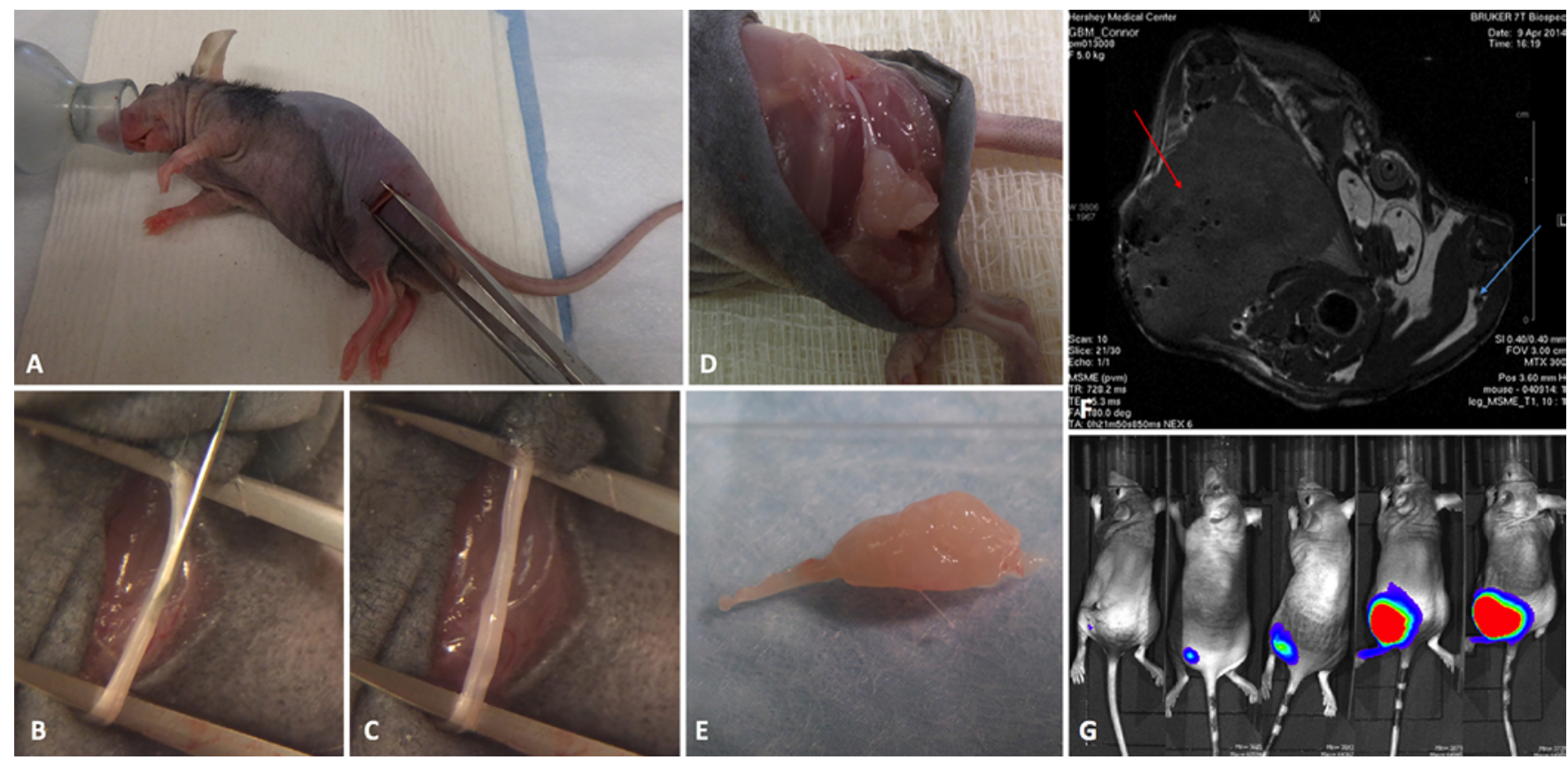

FIG. 1. Surgical technique for the orthoxenograft MPNST model. A: The mouse was anesthetized with isoflurane, and an incision was made parallel to the femur. Muscle planes were divided, and the sciatic nerve was isolated. B: Intraneural injection of 50,000 STS26T luciferase-transfected cells in $5 \mu$ l was performed under magnification to minimize efflux of the cell suspension into surrounding tissues. C: After injection, the nerve appeared swollen and pink due to the presence of intraneural tumor cells and media. D: Postmortem examination revealed a well-circumscribed tumor continuous with the sciatic nerve. E: The tumor was in continuity with the sciatic nerve after excision. Imaging confirmed the orthoxenograft MPNST model. F: An MR image of mouse hindlimbs obtained 4 weeks after intraneural tumor cell injection demonstrates MPNST (red arrow) and control sciatic nerve (blue arrow). STS26T cells were transfected with luciferase and were thus able to be imaged using IVIS after luciferin injection. These representative images demonstrate tumor progression throughout 4 weeks in an untreated mouse. G (left to right): IVIS confirmation of tumor progression 1 day, 1 week, 2 weeks, 3 weeks, and 4 weeks after intraneural injection of MPNST cells. Figure is available in color online only. 
Next, a subcutaneous injection of $100 \mu \mathrm{l}$ of D-Luciferin Substrate (purchased from Caliper LS and diluted in 35 $\mathrm{ml}$ of deionized $\mathrm{H}_{2} \mathrm{O}$ with a final concentration of 28.57 $\mathrm{mg} / \mathrm{ml}$ ) was administered. These mice were weighed and then transferred to the imaging chamber, where anesthesia was maintained with a concentration of $1 \%-2 \%$ isoflurane emitted through nose cones. Five minutes postinjection of D-Luciferin, imaging using the IVIS 50 (PerkinElmer) was performed according to the manufacturer's protocol. The IVIS was run for 1 minute and bioluminescence was recorded. The imaging was considered positive for tumor if there was a measureable amount of bioluminescence. If no signal was seen, reimaging was performed by immediately running the IVIS for 1 more minute. If no signal was seen on multiple repeat images, the imaging was considered negative for tumor. Imaging was performed on days $3,7,14,21,28,35$, and 42 after tumor cell injection. Confirmation of tumor progression is shown by MRI (Fig. 1F) and IVIS (Fig. 1G-K).

\section{Treatment of Nerve Tumors}

After it was confirmed that there was a measurable signal from the tumor cells, the intensity of bioluminescence was measured using Living Image software. All values were ranked in order of bioluminescent signal intensity and then evenly distributed into 3 homogeneous groups: 1 control group and 2 treatment groups.

The control group mice $(\mathrm{n}=13)$ received intraperitoneal (IP) injections of PBS equal to the volume used in the second treatment group and at the same intervals. The first treatment group $(\mathrm{n}=13)$ received MLN8237 (Takeda Pharmaceuticals) via daily oral gavage $(30 \mathrm{mg} / \mathrm{kg}$ in 100 $\mu \mathrm{l}$ of a $1 \%$ bicarbonate and $10 \%$ 2-hydroxypropyl betacyclodextrin solution) similar to previous studies. ${ }^{28,33,34}$ The second treatment group received IP injections of ifosfamide $(60 \mathrm{mg} / \mathrm{kg}$ in $300 \mu \mathrm{l}$ of PBS) for the first 3 days of treatment and IP injections of doxorubicin $(4 \mathrm{mg} / \mathrm{kg}$ in 100 $\mu \mathrm{l}$ of PBS) at weekly intervals..$^{19,40}$ Weights were assessed and recorded each week to assess for toxicity of treatment. Mouse weights from all groups remained consistent throughout the study, demonstrating minimal toxicity from treatments (data not shown). We chose to treat for approximately 1 month (32 days) based on previous studies assessing the efficacy of MLN8237 in vivo as well as published data on the pharmacodynamics of the drug..$^{28,33}$

\section{Statistical Analysis}

All statistical analysis was done using R. A power analysis using data from a pilot study was performed $(\alpha=$ $0.05, \beta=0.2$ ). Based on this, we chose to include 42 mice (14 per group). Baseline imaging characteristics of each group were compared using 1-way ANOVA. This showed no initial statistically significant difference in tumor bioluminescence between groups, demonstrating successful stratification. The average bioluminescence of each group at each interval was compared using 1-way ANOVA. A Tukey honest significant difference post hoc test was then used to assess for specific differences between groups. A linear regression was performed on all collected data points by group to assess for rate of tumor growth. The slopes of the lines between treatment groups were then compared using a 2-tailed, unpaired t-test (level of significance $=0.05$ ). Last, a survival analysis was performed using Kaplan-Meier curves. The curves were compared by log-rank test. A Cox proportional hazards model was also used to generate a hazard ratio.

\section{Results Immunohistochemistry of Aurora Kinase in the STS26T Cell Line}

In Fig. 2A-C, we demonstrate that the STS26T cells express robust signal of the oncogene aurora kinase A, corroborating previous studies. ${ }^{23-25}$ Next, to test whether inhibition of the high levels of aurora kinase by MLN8237 causes significant cell death, in vitro cytotoxicity studies were performed as shown in Fig. 2D.

\section{Cytotoxicity of MLN8237 in Cell Culture}

Four thousand STS26T cells per well were plated in 96-well plates in quintuplicate and allowed to adhere overnight. MLN8237 was then added to the wells in increasing dosage and allowed to incubate for 48 hours. After 48 hours, cell death analysis was performed (Fig. 2D). Compared with control samples, a dosage of $1 \mathrm{nM}$ MLN8237 caused 1\% cell death, $10 \mathrm{nM}$ MLN8237 caused 9\% cell death, $100 \mathrm{nM}$ MLN8237 caused 53\% cell death, and 1000 nM MLN8237 caused 67\% cell death. The efficacy of the cell culture model warranted in vivo studies of tumor progression and growth.

\section{Analysis of Tumor Growth}

Forty-two mice were injected with the STS26T-Luc cell line, 40 of which were confirmed to have tumors. As discussed above, mice were divided into 3 homogeneous groups and each group was randomly assigned as the control, MLN8237, or doxorubicin/ifosfamide group. Imaging was performed and tumor bioluminescence was recorded. These data are summarized in Fig. 3A-O, which shows decreased tumor burden of the MLN8237 group compared with the control and doxorubicin/ifosfamide groups toward the end of the study.

Overall tumor counts were quantified and recorded. The difference in mean bioluminescence was compared between groups (Table 1). These values were also plotted with confidence intervals in Fig. 3P-R. Figure 3P shows tumor counts throughout the length of the study, whereas the images in Fig. 3Q and R split the tumor counts into 3 -week blocks. This was done because the tumor counts toward the end of the study were extremely high, increasing the $y$-axis and obscuring differences that were seen between groups at earlier time points (e.g., 21 days).

To further delineate the relationship between the change in tumor bioluminescence and time according to the treatment group, we performed a linear regression analysis and compared the slopes of the regression lines using a 2-tailed, unpaired t-test. The results of this analysis are shown in Fig. 4 (left).

\section{Survival Analysis}

A survival analysis was performed using Kaplan-Meier 

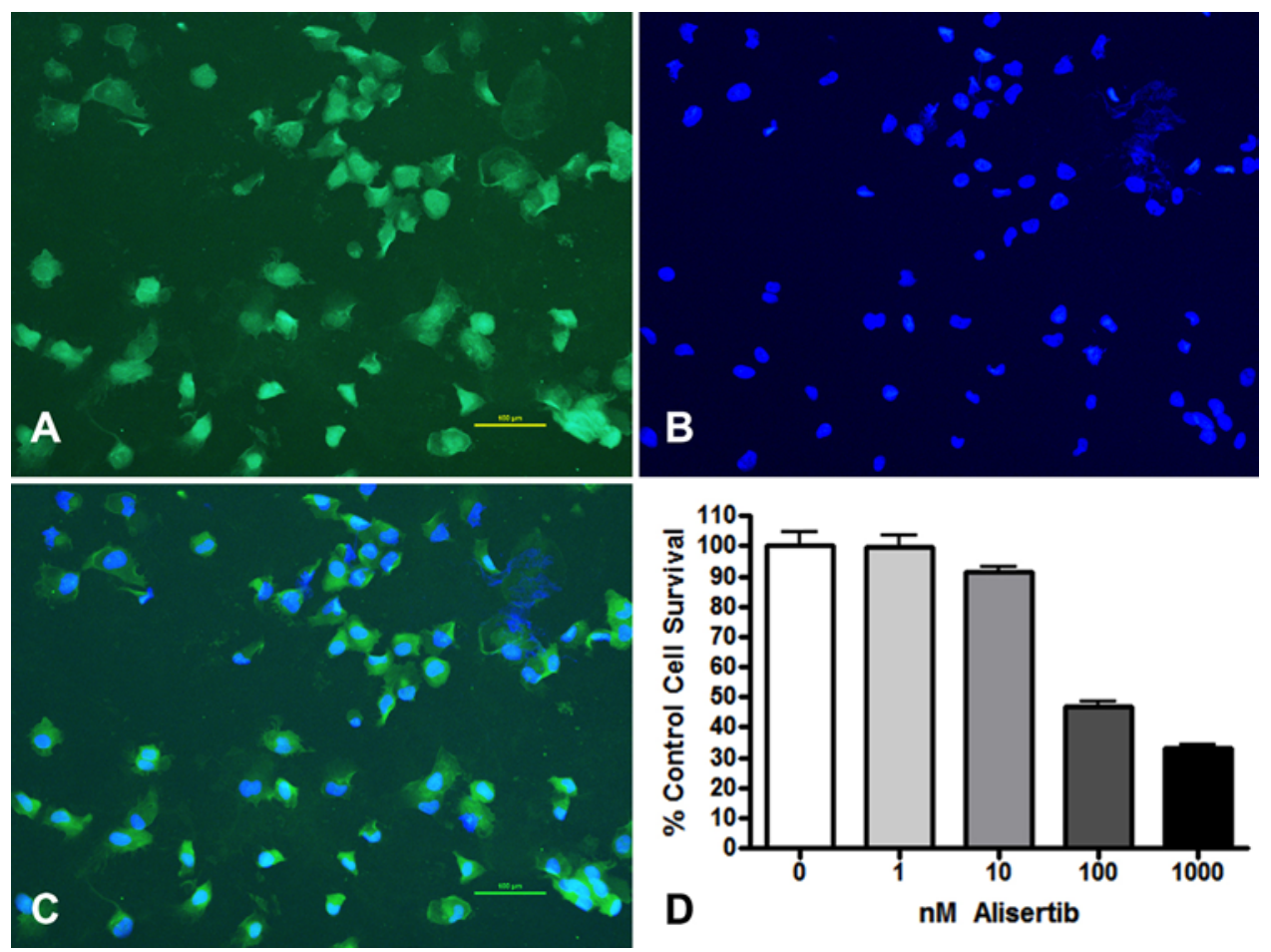

FIG. 2. Immunohistochemistry of aurora kinase A in STS26T MPNST cells. A: Robust expression of aurora kinase $A$ is seen in the STS26T cells. B: DAPI staining of cell nuclei. Original magnification $\times 40$. C: Merged images of aurora kinase A and DAPI in STS26T MPNST cells. D: Cytotoxicity of STS26T cells exposed to increasing concentrations of MLN8237 in vitro. Compared with control samples, a dosage of $1 \mathrm{nM} \mathrm{MLN8237} \mathrm{caused} \mathrm{1 \%} \mathrm{cell} \mathrm{death,} 10 \mathrm{nM}$ MLN8237 caused 9\% cell death, $100 \mathrm{nM} \mathrm{MLN8237}$ caused $53 \%$ cell death, and 1000 nM MLN8237 caused $67 \%$ cell death. Figure is available in color online only.
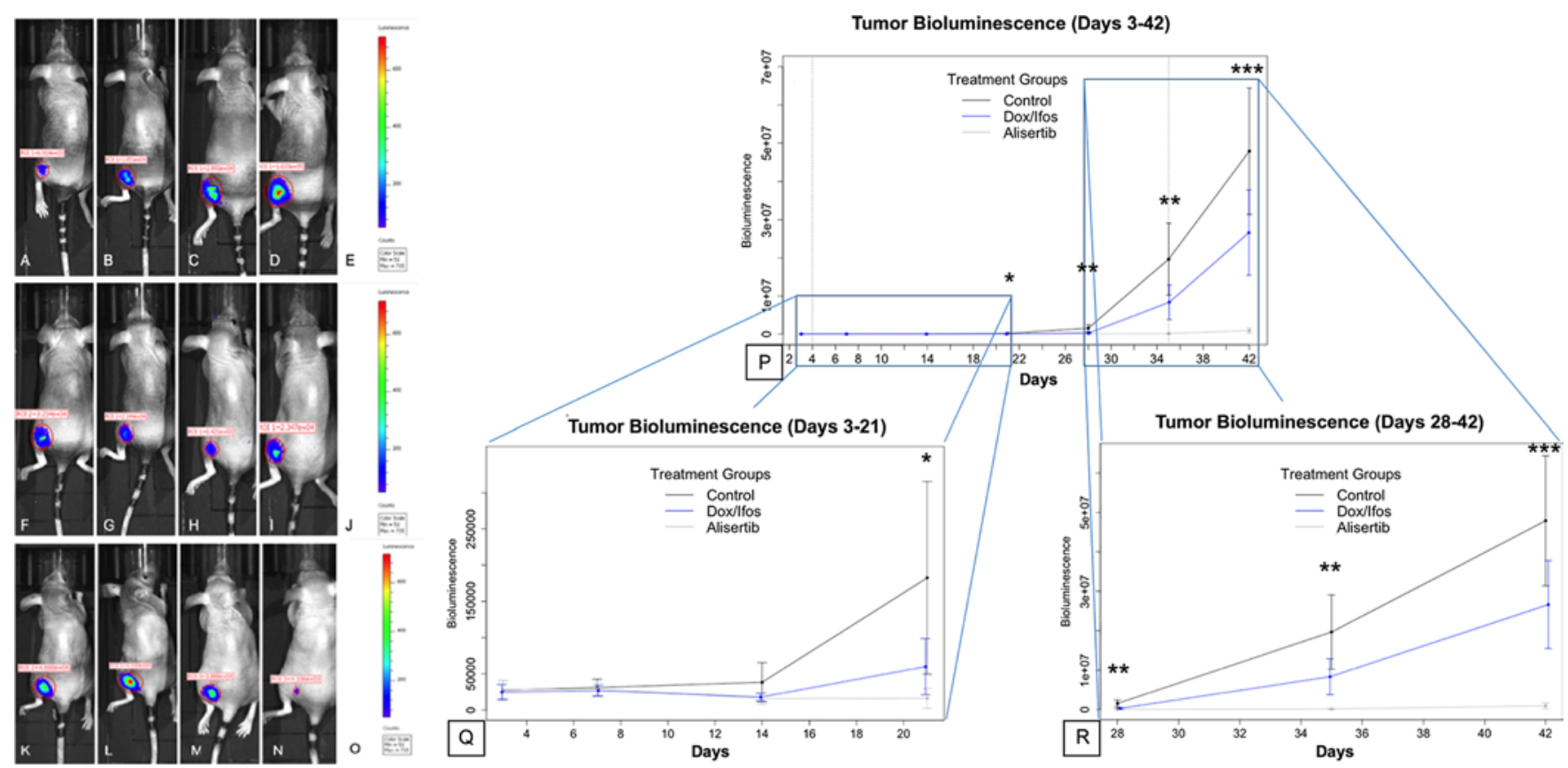

FIG. 3. Representative images of tumor progression over time. The control group (A-D), doxorubicin/ifosfamide-treated group (F-I), MLN8237-treated group (K-N), and bioluminescence scales (E, J, and O) are shown. Mice were injected on day 0 and the first imaging was performed on day 3. Treatment was given from days 4 to 35 (shown by dotted lines in panel P). Imaging was done at weekly intervals. Q: Mean tumor counts by group were recorded from days 3 to 21 . R: Average tumor counts by group were recorded from days 28 to 35 . Treatment was stopped at day 35. At day 42, a statistically significant difference between the MLN8237 and doxorubicin/ifosfamide groups was observed $(p=0.036)$. Dox $=$ doxorubicin; ifos = ifosfamide. ${ }^{*} p<0.05$; ${ }^{* *} p<0.01$; ${ }^{* * *} p<0.001$. Figure is available in color online only. 


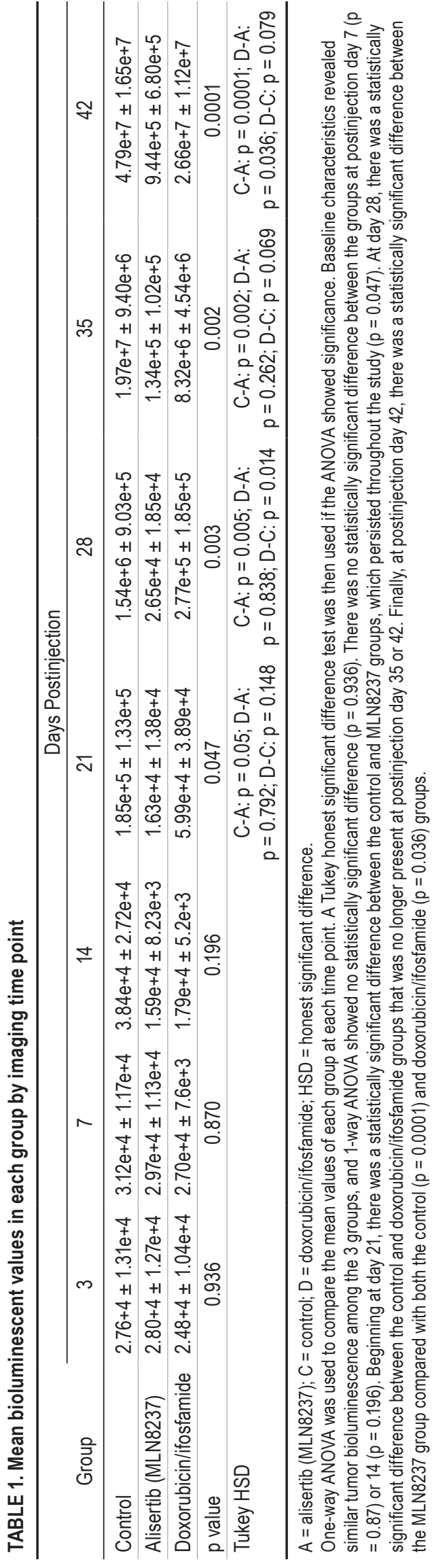

curves (Fig. 4 right). Two mice were lost in the MLN8237 group due to complications with oral gavage at the beginning of the study. These mice had a collection of blood in the mediastinum, presumably from esophageal rupture, and were thus censored. Median survival times were calculated and found to be longer in the MLN8237 group than the other 2 groups. A Cox proportional hazards model was used to calculate and compare hazard rates between the treatment groups. When doxorubicin/ifosfamide was compared with MLN8237, the hazard ratio was 2.945 (95\% CI $1.251-6.935 ; \mathrm{p}=0.0134)$.

\section{Immunohistochemistry of Tumor Samples}

Mice from each group were killed and tumor samples were frozen. Immunohistochemical analysis of tumor tissue for markers of tumor growth, proliferation, and apoptosis was performed (Fig. 5). Ki-67 cellular proliferation marker analysis demonstrated decreasing levels in doxorubicin/ifosfamide- and MLN8237-treated tumors compared with control. Cleaved caspase-3 apoptotic marker increased in both doxorubicin/ifosfamide- and MLN8237treated tumors. Cyclin B1 cellular proliferation marker analysis demonstrated decreasing levels in doxorubicin/ ifosfamide- and MLN8237-treated tumors compared with the control group. DAPI staining (40x) was then performed to assess for enlargement of the nucleus, which may demonstrate that MLN8237 successfully inhibited aurora kinase and halted the cell cycle at $\mathrm{M}$ phase, leading to cell death, as well as polyploidy due to inhibition of aurora kinase, leading to many nondisjunction events.

\section{Discussion}

MPNSTs are uncommon, which makes human studies difficult, expensive, and time consuming. This accentuates the need for translationally accurate, robust, and cost-effective preclinical models. A major goal of this study was to produce such a model and evaluate its response to novel therapies. The development of an orthoxenograft MPNST sciatic model circumvents drawbacks of other models by creating a robust tumor in a physiologically relevant microenvironment, allowing for accurate and practical tumor burden quantification through luciferin fluorescence.

This study provides 2 novel and significant findings. First, we established an orthoxenograft murine model using a non-NF1 cell line. Second, we demonstrated the efficacy of an aurora kinase inhibitor.

\section{Preclinical MPNST Model}

\section{Limitations of Previous Models}

Murine models for MPNST reported in the literature can be divided into 2 broad categories: transgenic and xenograft. Transgenic murine models rely on the alteration of the mouse genome to promote tumor growth. Xenograft models use human tumor cells that are directly grafted into immunodeficient mice. Several types of xenograft models exist. The orthoxenograft murine model uses human tumor cells grafted into their tissue of origin (usually the sciatic nerve). Ectopic xenograft models graft human tumor cells into a location other than their tissue of origin (often subcutaneous tissues). 

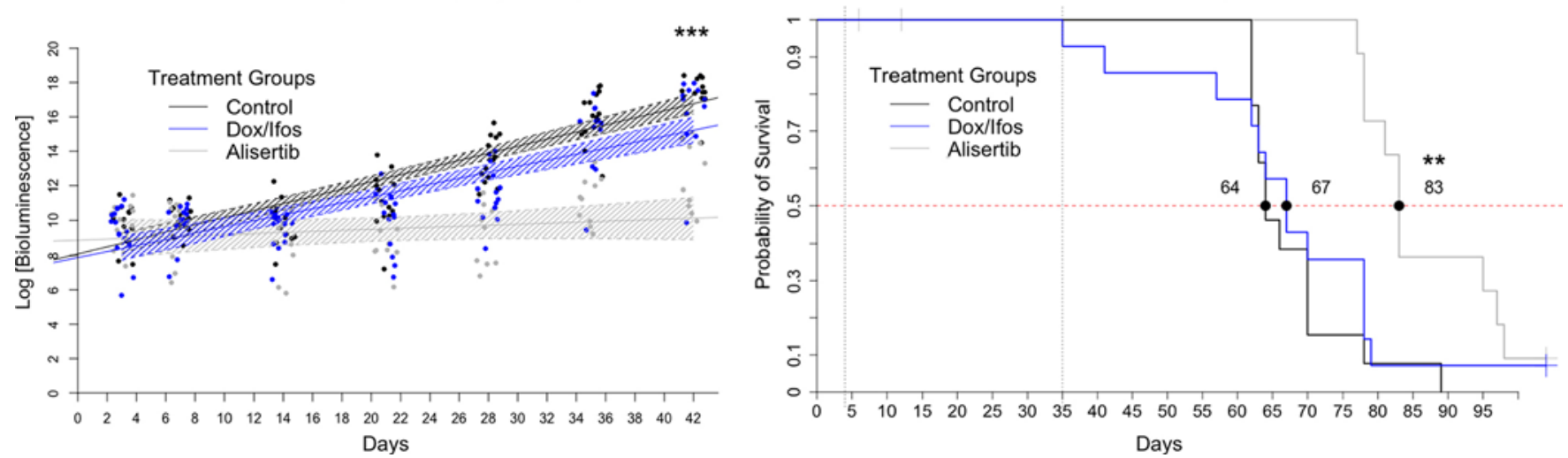

FIG. 4. Tumor rate of growth and survival analysis. Left: Scatter plot shows a linear regression (days 3-42) through the individual bioluminescent values of the mice at each time point (control = black, doxorubicin/ifosfamide = blue, MLN8237 = gray). The y-axis is log [bioluminescence] and the $x$-axis is postinjection days. The shaded areas represent the confidence intervals for each line. Right: Kaplan-Meier survival curves for each of the experimental groups. The control group is represented by the black line, the MLN8237-treated group by the gray line, and the doxorubicin/ifosfamide-treated group by the blue line. The 2 mice that died as the result of oral gavage were censored; this is represented by the vertical hash marks on the MLN8237 survival curve. The vertical gray dotted lines show treatment start and end dates (postinjection days 4 and 35). The red horizontal dotted line intersects the $y$-axis at the point of $50 \%$ survival. The median survival of each group is marked by the intersection of the $50 \%$ survival line and the survival curve (black dots). The median survival times were 64,67 , and 83 days for the control, doxorubicin/ifosfamide, and MLN8237 groups, respectively. All of the control mice had died by day 90 , whereas 1 of the doxorubicin/ifosfamide-treated mice lived until the conclusion of the study. On inspection, this mouse had a moderate-sized tumor in the hindlimb. One mouse in the MLN8237 group survived until the conclusion of the study. On inspection, no tumor could be visualized. When compared using a log-rank test, the curves were statistically significantly different $(p=0.003) .{ }^{*} p<0.05 ;{ }^{* *} p<0.01 ;{ }^{* * *} p<0.001$. Figure is available in color online only.

(A) Ki-67
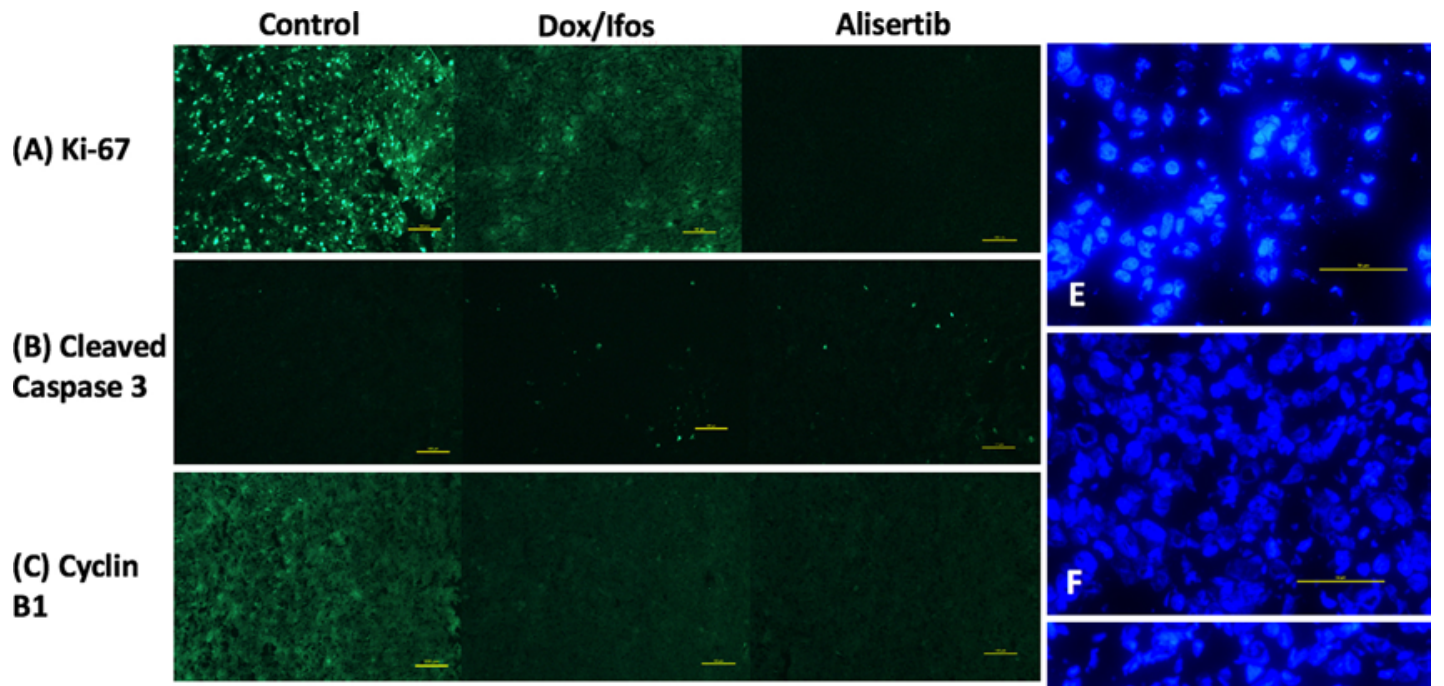

\section{(B) Cleaved Caspase 3}

\section{(D) Aurora} Kinase A
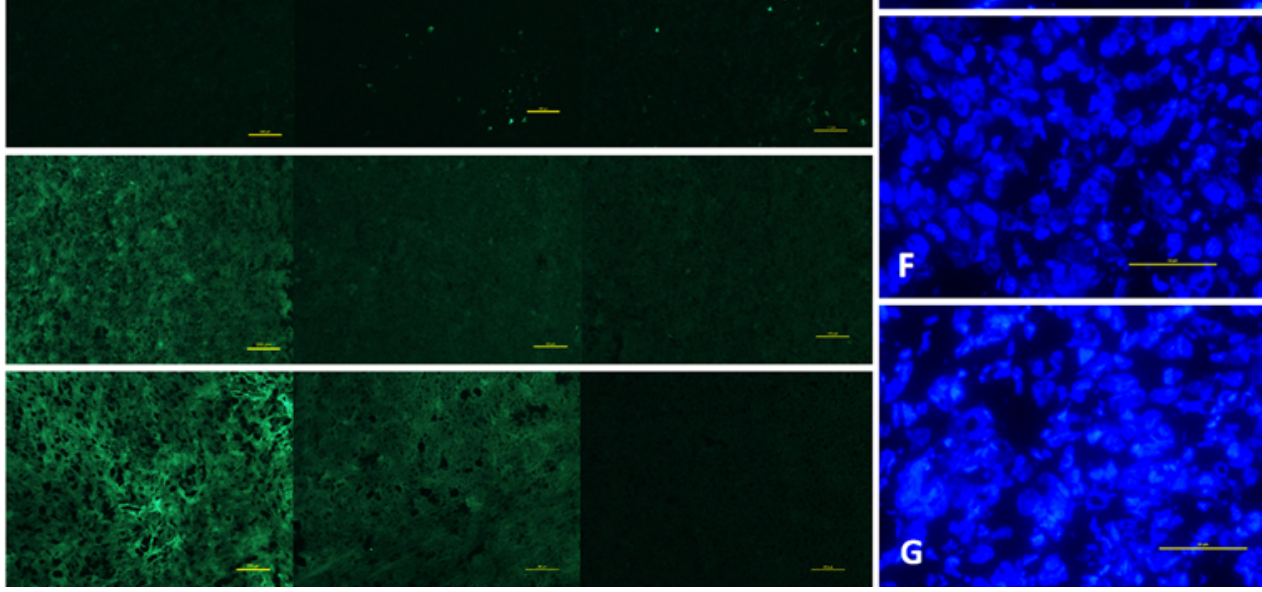

FIG. 5. Immunohistochemical analysis of tumor tissue for markers of tumor growth, proliferation, and apoptosis. A: A Ki-67 cellular proliferation marker demonstrated decreasing levels in doxorubicin/ifosfamide- and MLN8237-treated tumors compared with the control group. B: Cleaved caspase-3 apoptotic marker was increased in both doxorubicin/ifosfamide- and MLN8237-treated tumors. C: Cyclin B1 cellular proliferation marker demonstrated decreasing levels in doxorubicin/ifosfamide- and MLN8237treated tumors compared with the control group. D: Aurora kinase A was shown to be increased in the control tumors, whereas its staining decreased in the doxorubicin/ifosfamide group and ceased in the MLN8237 samples. E-G: DAPI staining was performed to assess for changes in the appearance of nuclei. The MLN8237 group showed enlargement and an abnormal appearance of nuclei and polyploidy. Control $(E)$, doxorubicin $(F)$, and MLN8237 (G). Original magnification $\times 40$. Figure is available in color online only. 
Several problems have been encountered with previous models. Transgenic models have been successfully used in the evaluation of MPNST therapies, 3,9,44,46 but have inherent drawbacks associated with them. One serious problem is the inability to determine when and where tumors will develop, making it difficult to time potential therapies. Furthermore, it is thought that there may also be alterations in other cellular pathways associated with transgenic expression. ${ }^{24,50}$

Likewise, xenograft models have their own drawbacks. The first difficulty has been creating an accurate model with orthotopic tumor growth. Previous studies have relied on subcutaneous injection of tumor cells. ${ }^{8,20-22,27}$ Although reproducible and inexpensive, this type of model places tumor cells in an abnormal microenvironment, which may lead to a false-positive response to the therapy being evaluated. Significant site-specific variation in response to therapy has been reported in xenograft murine models, showing the importance of this microenvironment when evaluating the therapeutic efficacy of new treatments..$^{24,49}$

A second difficulty has been assessing response to therapy over time. This is especially true with orthotopic models in which tumor volume cannot be assessed using external measurements. Some studies have relied on MRI as a noninvasive means of measuring tumor growth. ${ }^{8,39}$ Other studies have relied on clinical examination and rating of neurological deficit using scoring systems. ${ }^{2}$ Other techniques include the assessment of postmortem tumor volume. Each of these methods of assessing tumor growth comes with its own difficulties. In our experience, MRI is expensive and time consuming, and it can result in high mortality of the animals due to the need for high doses of anesthesia. Neurological examination, although potentially clinically relevant, is an indirect measure of tumor growth and may not always correlate with tumor response to therapy.

A third difficulty has been developing an orthoxenograft model that does not require fresh tumor tissue. Several studies have used fresh tumor tissue minced into small pieces. These pieces are then placed in proximity to the sciatic nerve and tumor growth is assessed through various means. ${ }^{7,8}$ This model has some inherent limitations, such as the need for fresh tissue and the inability to transfect cells with luciferase for in vivo imaging (that is, using IVIS).

Our model addresses the drawbacks seen in previous models by directly controlling tumor location and growing tumors in their natural microenvironment. Although we controlled how many cells were injected and we stratified based on the size of tumors, there was still variation in sizes, which may have had a role in the efficacy of treatments. There is also an inherent operator-dependent variation and a learning curve when beginning the process of tumor cell injections.

\section{Orthoxenograft Murine Model Using Luciferase for In Vivo Imaging}

We present an orthoxenograft murine model using the STS26T MPNST cell line (non-NF1) transfected to constitutively express luciferase. The benefits of this type of model are significant. First, it is orthotopic and better rep- resents the true disease state of patients with MPNSTs, with tumor arising within neural structures and in a normal and physiologically relevant microenvironment. Second, it is reproducible and not labor intensive. Finally, it allows for the use of IVIS, which is less expensive and less time intensive than MRI and has been shown to accurately evaluate tumor volume in xenograft models. ${ }^{22,25,45,50} \mathrm{Un}-$ like previous studies, we developed a successful non-NF1 MPNST model while also evaluating tumor response to a novel therapy. ${ }^{50}$

\section{MLN8237 as a Treatment for MPNSTs}

MPNSTs are an aggressive soft-tissue sarcoma for which there are no good systemic therapies. Several MPNST cell lines (including STS26T) have shown increased expression of aurora kinase A, making it a potential target in cancer therapy. Patel et al. found that aurora kinase A (AURKA) was overexpressed and genomically amplified in MPNSTs. Administration of an aurora kinase A inhibitor (MLN8237) in vitro blocked cell growth, and in vivo administration increased survival time in mice. ${ }^{38}$ Mohan et al. confirmed the findings of Patel et al. and further solidified the potential of aurora kinase inhibition in MPNST therapy. ${ }^{33}$

Herein, we present a preclinical study that assessed the response of a human non-NF1 MPNST cell line in an orthoxenograft animal model to MLN8237 compared with best medical therapy (doxorubicin and ifosfamide). Using IVIS, we were able to monitor tumor bioluminescence each week. Although the 2 treatment groups started with similar average bioluminescent signals, we saw that by posttreatment day 21 there was a statistically significant difference between the MLN8237 and control groups, which persisted until the end of the study. There was a single time point (postinjection day 28) when there was a statistically significant difference between the doxorubicin/ifosfamide and control groups. This statistically significant difference was lost at the end of the study (postinjection days 35 and 42). The loss of significance of the doxorubicin/ifosfamide group, but continued significance of the MLN8237 group compared with the control group, demonstrates the durable response of MLN8237 on MPNSTs. Even more striking is the fact that, by postinjection day 42 (1 week after cessation of treatment), there was a statistically significant difference between the MLN8237 group and the gold standard chemotherapy group (doxorubicin/ifosfamide) ( $p$ $=0.036$ ).

Survival analysis using Kaplan-Meier curves correlated with the in vivo imaging results and showed a statistically significant survival advantage in the group that was treated with MLN8237 ( $p=0.007)$. The median survival was 83 days in the MLN8237 group compared with 64 and 67 days in the control and doxorubicin/ifosfamide groups, respectively. A comparison of hazard rates between the doxorubicin/ifosfamide and MLN8237 groups revealed a hazard ratio of 2.945, again confirming the benefit that MLN8237 conferred on survival in our study $(\mathrm{p}=0.0134)$.

For confirmation of the effects seen in vivo, immunohistochemical analysis of the in vivo tumors was performed. This demonstrated decreasing levels of cellular proliferation markers Ki-67 and cyclin B1 and increasing 
levels of the apoptosis marker cleaved caspase-3, all of which corroborate the decrease in tumor progression of the treated groups compared with the control group. A biomarker for patients who are treated with MLN8237 would also be desirable. We showed that levels of aurora kinase A decreased to negligible amounts in MLN8237-treated tumors, which may demonstrate the potential for aurora kinase $\mathrm{A}$ inhibition to be a biomarker for treatment efficacy in patients being treated with MLN8237. The mitotic effects of inhibition of aurora kinase A were also analyzed. DAPI staining (40x) showed abnormal enlarged nuclei in MLN8237-treated tumors, which may demonstrate that MLN8237 successfully inhibited aurora kinase and halted the cell cycle at $\mathrm{M}$ phase, leading to cell death, as well as polyploidy due to nondisjunction events.

Unlike previous studies, our study demonstrates the effectiveness of using MLN8237 to treat MPNSTs in an accurate and reproducible orthoxenograft model with longitudinal in vivo monitoring of tumor progression. This suggests that MLN8237 may be successfully incorporated into the treatment of MPNSTs and should be further investigated. A recent phase II study evaluating the efficacy of MLN8237 in advanced/metastatic sarcoma showed that it was generally tolerated well by patients. Although the study did not reach its primary end point of overall response and the sample size was small, it did show encouraging progression-free survival at 12 weeks $(60 \%$ in the MPNST group). ${ }^{10}$ These progression-free survival results corroborate the durable response observed in our study and demonstrate the potential for MLN8237 treatment in MPNSTs. Further studies are needed to show the benefit of MLN8237 for treating patients with MPNSTs; however, the current data are promising.

\section{Conclusions}

A growing body of literature shows that aurora kinase inhibition may be beneficial in the treatment of MPNSTs. The results of our study demonstrate that MLN8237 is superior to combination treatment with doxorubicin and ifosfamide in a preclinical orthoxenograft animal model. Our data have major implications for the future of MPNST research by providing a robust murine model as well as evidence that MLN8237 may be an effective treatment for MPNSTs. Given the success of MLN8237 in this MPNST cell line, we plan to investigate its potential role in the treatment of benign nerve sheath tumors. This may provide a nonsurgical treatment for those suffering from NF1 for whom there is a high risk of malignant transformation of neurofibromas into MPNSTs. Finally, to truly characterize its effectiveness in humans, a multicenter clinical trial of MLN8237 is necessary.

\section{Acknowledgments}

Takeda Pharmaceuticals provided the alisertib compound.

\section{References}

1. Anghileri M, Miceli R, Fiore M, Mariani L, Ferrari A, Mussi $\mathrm{C}$, et al: Malignant peripheral nerve sheath tumors: prognostic factors and survival in a series of patients treated at a single institution. Cancer 107:1065-1074, 2006
2. Antoszczyk S, Spyra M, Mautner VF, Kurtz A, StemmerRachamimov AO, Martuza RL, et al: Treatment of orthotopic malignant peripheral nerve sheath tumors with oncolytic herpes simplex virus. Neuro Oncol 16:1057-1066, 2014

3. Brosius SN, Turk AN, Byer SJ, Brossier NM, Kohli L, Whitmire A, et al: Neuregulin-1 overexpression and Trp53 haploinsufficiency cooperatively promote de novo malignant peripheral nerve sheath tumor pathogenesis. Acta Neuropathol 127:573-591, 2014

4. Carli M, Ferrari A, Mattke A, Zanetti I, Casanova M, Bisogno G, et al: Pediatric malignant peripheral nerve sheath tumor: the Italian and German soft tissue sarcoma cooperative group. J Clin Oncol 23:8422-8430, 2005 (Erratum in J Clin Oncol 24:724, 2006)

5. Carmena M, Earnshaw WC: The cellular geography of aurora kinases. Nat Rev Mol Cell Biol 4:842-854, 2003

6. Casali PG, Jost L, Sleijfer S, Verweij J, Blay JY: Soft tissue sarcomas: ESMO clinical recommendations for diagnosis, treatment and follow-up. Ann Oncol 19 (Suppl 2):ii89-ii93, 2008

7. Castellsagué J, Gel B, Fernández-Rodríguez J, Llatjós R, Blanco I, Benavente Y, et al: Comprehensive establishment and characterization of orthoxenograft mouse models of malignant peripheral nerve sheath tumors for personalized medicine. EMBO Mol Med 7:608-627, 2015

8. Chang LS, Jacob A, Lorenz M, Rock J, Akhmametyeva EM, Mihai G, et al: Growth of benign and malignant schwannoma xenografts in severe combined immunodeficiency mice. Laryngoscope 116:2018-2026, 2006 (Erratum in Laryngoscope 116:2236, 2006)

9. Chau V, Lim SK, Mo W, Liu C, Patel AJ, McKay RM, et al: Preclinical therapeutic efficacy of a novel pharmacologic inducer of apoptosis in malignant peripheral nerve sheath tumors. Cancer Res 74:586-597, 2014

10. Dickson MA, Mahoney MR, Tap WD, D'Angelo SP, Keohan ML, Van Tine BA, et al: Phase II study of MLN8237 (Alisertib) in advanced/metastatic sarcoma. Ann Oncol 27:1855-1860, 2016

11. Du X, Yang J, Ylipää A, Zhu Z: Genomic amplification and high expression of EGFR are key targetable oncogenic events in malignant peripheral nerve sheath tumor. J Hematol Oncol 6:93, 2013

12. Ducatman BS, Scheithauer BW, Piepgras DG, Reiman HM, Ilstrup DM: Malignant peripheral nerve sheath tumors. A clinicopathologic study of 120 cases. Cancer 57:2006-2021, 1986

13. Evans DGR, Baser ME, McGaughran J, Sharif S, Howard E, Moran A: Malignant peripheral nerve sheath tumours in neurofibromatosis 1. J Med Genet 39:311-314, 2002 (Erratum in J Med Genet 40:304, 2003)

14. Farid M, Demicco EG, Garcia R, Ahn L, Merola PR, Cioffi A, et al: Malignant peripheral nerve sheath tumors. Oncologist 19:193-201, 2014

15. Ferrari A, Bisogno G, Carli M: Management of childhood malignant peripheral nerve sheath tumor. Paediatr Drugs 9:239-248, 2007

16. Gong M, Ma J, Li M, Zhou M, Hock JM, Yu X: MicroRNA-204 critically regulates carcinogenesis in malignant peripheral nerve sheath tumors. Neuro Oncol 14:1007-1017, 2012

17. Gupta G, Mammis A, Maniker A: Malignant peripheral nerve sheath tumors. Neurosurg Clin N Am 19:533-543, v, 2008

18. Hajdu SI: Peripheral nerve sheath tumors. Cancer 72:35493552, 1993

19. Hanly L, Figueredo R, Rieder MJ, Koropatnick J, Koren G: The effects of N-acetylcysteine on ifosfamide efficacy in a mouse xenograft model. Anticancer Res 32:3791-3798, 2012 
20. Hirokawa Y, Nakajima H, Hanemann CO, Kurtz A, Frahm S, Mautner V, et al: Signal therapy of NF1-deficient tumor xenograft in mice by the anti-PAK1 drug FK228. Cancer Biol Ther 4:379-381, 2005

21. Hirokawa Y, Nheu T, Grimm K, Mautner V, Maeda S, Yoshida M, et al: Sichuan pepper extracts block the PAK1/ cyclin D1 pathway and the growth of NF1-deficient cancer xenograft in mice. Cancer Biol Ther 5:305-309, 2006

22. Johansson G, Mahller YY, Collins MH, Kim MO, Nobukuni $\mathrm{T}$, Perentesis J, et al: Effective in vivo targeting of the mammalian target of rapamycin pathway in malignant peripheral nerve sheath tumors. Mol Cancer Ther 7:1237-1245, 2008

23. Kamran SC, Shinagare AB, Howard SA, Hornick JL, Ramaiya NH: A-Z of malignant peripheral nerve sheath tumors. Cancer Imaging 12:475-483, 2012

24. Killion JJ, Radinsky R, Fidler IJ: Orthotopic models are necessary to predict therapy of transplantable tumors in mice. Cancer Metastasis Rev 17:279-284, 1998-1999

25. Klerk CPW, Overmeer RM, Niers TMH, Versteeg HH, Richel DJ, Buckle T, et al: Validity of bioluminescence measurements for noninvasive in vivo imaging of tumor load in small animals. Biotechniques 43:7-13, 30, 2007

26. Kroep JR, Ouali M, Gelderblom H, Le Cesne A, Dekker TJA, Van Glabbeke M, et al: First-line chemotherapy for malignant peripheral nerve sheath tumor (MPNST) versus other histological soft tissue sarcoma subtypes and as a prognostic factor for MPNST: an EORTC soft tissue and bone sarcoma group study. Ann Oncol 22:207-214, 2011

27. Mahller YY, Vaikunth SS, Currier MA, Miller SJ, Ripberger MC, Hsu YH, et al: Oncolytic HSV and erlotinib inhibit tumor growth and angiogenesis in a novel malignant peripheral nerve sheath tumor xenograft model. Mol Ther 15:279-286, 2007

28. Manfredi MG, Ecsedy JA, Chakravarty A, Silverman L, Zhang M, Hoar KM, et al: Characterization of Alisertib (MLN8237), an investigational small-molecule inhibitor of aurora A kinase using novel in vivo pharmacodynamic assays. Clin Cancer Res 17:7614-7624, 2011

29. Marumoto T, Honda S, Hara T, Nitta M, Hirota T, Kohmura E, et al: Aurora-A kinase maintains the fidelity of early and late mitotic events in HeLa cells. J Biol Chem 278:5178651795, 2003

30. Marumoto T, Zhang D, Saya H: Aurora-A - a guardian of poles. Nat Rev Cancer 5:42-50, 2005

31. McGaughran JM, Harris DI, Donnai D, Teare D, MacLeod R, Westerbeek R, et al: A clinical study of type 1 neurofibromatosis in north west England. J Med Genet 36:197-203, 1999

32. Miller SJ, Rangwala F, Williams J, Ackerman P, Kong S, Jegga AG, et al: Large-scale molecular comparison of human Schwann cells to malignant peripheral nerve sheath tumor cell lines and tissues. Cancer Res 66:2584-2591, 2006

33. Mohan P, Castellsague J, Jiang J, Allen K, Chen H, Nemirovsky O, et al: Genomic imbalance of HMMR/ RHAMM regulates the sensitivity and response of malignant peripheral nerve sheath tumour cells to aurora kinase inhibition. Oncotarget 4:80-93, 2013

34. Nair JS, Schwartz GK: MLN-8237 : A dual inhibitor of aurora A and B in soft tissue sarcomas. Oncotarget 7:1289312903, 2016

35. Ng VY, Scharschmidt TJ, Mayerson JL, Fisher JL: Incidence and survival in sarcoma in the United States: a focus on musculoskeletal lesions. Anticancer Res 33:2597-2604, 2013

36. Palani S, Patel M, Huck J, Zhang M, Balani SK, Yang J, et al: Preclinical pharmacokinetic/pharmacodynamic/efficacy relationships for alisertib, an investigational small-molecule inhibitor of Aurora A kinase. Cancer Chemother Pharmacol 72:1255-1264, 2013
37. Pasmant E, Luscan A, Varin J, Laurendeau I, Parfait B, Vidaud D: Relevance of MPNST cell lines as models for NF1 associated-tumors. J Neurooncol 114:353-355, 2013

38. Patel AV, Eaves D, Jessen WJ, Rizvi TA, Ecsedy JA, Qian MG, et al: Ras-driven transcriptome analysis identifies aurora kinase $\mathrm{A}$ as a potential malignant peripheral nerve sheath tumor therapeutic target. Clin Cancer Res 18:5020-5030, 2012

39. Perrin GQ, Li H, Fishbein L, Thomson SA, Hwang MS, Scarborough MT, et al: An orthotopic xenograft model of intraneural NF1 MPNST suggests a potential association between steroid hormones and tumor cell proliferation. Lab Investig 87:1092-1102, 2007

40. Pervaiz N, Colterjohn N, Farrokhyar F, Tozer R, Figueredo A, Ghert M: A systematic meta-analysis of randomized controlled trials of adjuvant chemotherapy for localized resectable soft-tissue sarcoma. Cancer 113:573-581, 2008

41. Rodriguez FJ, Folpe AL, Giannini C, Perry A: Pathology of peripheral nerve sheath tumors: diagnostic overview and update on selected diagnostic problems. Acta Neuropathol 123:295-319, 2012

42. Sane KM, Mynderse M, Lalonde DT, Dean IS, Wojtkowiak JW, Fouad F, et al: A novel geranylgeranyl transferase inhibitor in combination with lovastatin inhibits proliferation and induces autophagy in STS-26T MPNST cells. J Pharmacol Exp Ther 333:23-33, 2010

43. Scaife CL, Pisters PW: Combined-modality treatment of localized soft tissue sarcomas of the extremities. Surg Oncol Clin N Am 12:355-368, 2003

44. Shollar D, Del Valle L, Khalili K, Otte J, Gordon J: JCV T-antigen interacts with the neurofibromatosis type 2 gene product in a transgenic mouse model of malignant peripheral nerve sheath tumors. Oncogene 23:5459-5467, 2004

45. Szentirmai O, Baker CH, Lin N, Szucs S, Takahashi M, Kiryu S, et al: Noninvasive bioluminescence imaging of luciferase expressing intracranial U87 xenografts: correlation with magnetic resonance imaging determined tumor volume and longitudinal use in assessing tumor growth and antiangiogenic treatment effect. Neurosurgery 58:365-372, 2006

46. Tanaka K, Eskin A, Chareyre F, Jessen WJ, Manent J, NiwaKawakita M, et al: Therapeutic potential of HSP90 inhibition for neurofibromatosis type 2. Clin Cancer Res 19:38563870,2013

47. Thway K, Fisher C: Malignant peripheral nerve sheath tumor: pathology and genetics. Ann Diagn Pathol 18:109-116, 2014

48. Trojanowski JQ, Kleinman GM, Proppe KH: Malignant tumors of nerve sheath origin. Cancer 46:1202-1212, 1980

49. Tsai MJ, Chang WA, Huang MS, Kuo PL: Tumor microenvironment: a new treatment target for cancer. ISRN Biochem 2014:351959, 2014

50. Turk AN, Byer SJ, Zinn KR, Carroll SL: Orthotopic xenografting of human luciferase-tagged malignant peripheral nerve sheath tumor cells for in vivo testing of candidate therapeutic agents. J Vis $\operatorname{Exp}$ (49):2558, 2011

51. Valentin T, Le Cesne A, Ray-Coquard I, Italiano A, Decanter G, Bompas E, et al: Management and prognosis of malignant peripheral nerve sheath tumors: the experience of the French Sarcoma Group (GSF-GETO). Eur J Cancer 56:77-84, 2016

52. Widemann BC: Current status of sporadic and neurofibromatosis type 1-associated malignant peripheral nerve sheath tumors. Curr Oncol Rep 11:322-328, 2009

53. Wong WW, Hirose T, Scheithauer BW, Schild SE, Gunderson LL: Malignant peripheral nerve sheath tumor: analysis of treatment outcome. Int J Radiat Oncol Biol Phys 42:351360, 1998

54. Yang J, Ylipää A, Sun Y, Zheng H, Chen K, Nykter M, et al: Genomic and molecular characterization of malignant peripheral nerve sheath tumor identifies the IGF1R pathway 
as a primary target for treatment. Clin Cancer Res 17:75637573,2011

55. Zehou O, Fabre E, Zelek L, Sbidian E, Ortonne N, Banu E, et al: Chemotherapy for the treatment of malignant peripheral nerve sheath tumors in neurofibromatosis 1: a 10-year institutional review. Orphanet J Rare Dis 8:127, 2013

\section{Disclosures}

The authors report no conflict of interest concerning the materials or methods used in this study or the findings specified in this paper.

\section{Author Contributions}

Conception and design: Payne, Mrowczynski, Slagle-Webb, Aregawi, Madhankumar, Harbaugh, Connor, Rizk. Acquisition of data: Payne, Mrowczynski, Slagle-Webb, Bourcier, Mau, Rizk. Analysis and interpretation of data: Payne, Mrowczynski,
Aregawi, Madhankumar, Harbaugh, Connor, Rizk. Drafting the article: Payne, Mrowczynski, Madhankumar, Harbaugh, Connor, Rizk. Critically revising the article: Payne, Mrowczynski, Harbaugh, Connor, Rizk. Reviewed submitted version of manuscript: Payne, Mrowczynski, Harbaugh. Statistical analysis: Payne, Mrowczynski, Connor, Rizk. Administrative/technical/material support: Slagle-Webb, Bourcier, Mau, Madhankumar, Lee, Rizk. Study supervision: Slagle-Webb, Madhankumar, Rizk.

\section{Supplemental Information}

Previous Presentations

Portions of this work were presented in poster form at the 21st Society for Neuro-Oncology Annual Scientific Meeting, Scottsdale, AZ, November 17-20, 2016.

\section{Correspondence}

Russell Payne: Penn State Milton S. Hershey Medical Center, Hershey, PA.rpayne@hmc.psu.edu. 